\title{
Is the spatially averaged spectrum equal to the global spectrum?
}

\author{
G. Pistone*, O. Di Stefano, S. Savasta, and R. Girlanda \\ INFM and Dipartimento di Fisica della Materia e Tecnologie Fisiche Avanzate, Università di Messina, \\ Salita Sperone 31, 98166 Messina, Italy
}

\begin{abstract}
Near-field optical spectroscopy and microscopy give access to excitations that cannot be revealed in the far zone. In order to investigate these remarkable differences, we present a theoretical analysis of the local optical properties of semiconductor quantum wells including the effects of disorder arising from interface fluctuations. The far-field absorption spectrum is compared with spatially averaged absorption spectra calculated at different spatial resolutions. We find that summing up local optical spectra does not reproduce the global spectrum in contrast to findings at diffraction-limited resolutions.
\end{abstract}

Near field microscopy and spectroscopy provide direct information on the spatial and energy distribution of light emitting nanometric centers [1-5] of semiconductor quantum structures. Moreover near field spectroscopy offers unique attributes in addition to high spatial resolution which might be explored in future experiments. For instance, the presence of optical fields with high lateral spatial frequencies able to excite surface states with high k-vectors not accessible by far-field optical excitations allows the possibility to confine the optical excitation to a very small volume below the diffraction limit. As a consequence optical spectra of homogeneous surface systems can display remarkable differences in the near and far zones [6,7]. These spectral changes are caused by the loss of evanescent modes (corresponding to modes with high lateral spatial frequencies) in the far zone. They have been analyzed theoretically in ideal disorder-free surface systems where in-plane k-vectors are good quantum numbers.

Here we analyze, in a realistic surface system affected by disorder effects, these additional opportunities of near-field spectroscopy. To what extent these spectral changes are detectable in real systems affected by disorder and imperfections? Are spatially averaged near-field spectra equal to far-field spectra? In order to isolate spectral changes due to excitation of states not accessible by far-field optical probes, we compare far-field absorption spectra with spatially averaged absorption spectra [8]. These averaged spectra are composed summing up local spectra obtained centering the confined illuminating beams on a fine mesh of points on the quantum well plane and they are calculated at different spatial resolutions. Our analysis is performed on quantum wells (QWs). Interface fluctuations in QWs result in an effective 2D (two-dimensional) spatially correlated random potential that tends to localize the center of mass (COM) motion of excitons $[9,10]$ and produces an inhomogeneous Gaussian-like absorption line $[11,12]$. Inhomogeneities and disorder effects give rise to surface quantum states, eventually localized, with mixed in-plane $k$-vectors (the in-plane $k$-vector is no more a good quantum number).

\footnotetext{
${ }^{*}$ Corresponding author: e-mail: Pistone@unime.it
} 
The total absorbed power under local illumination is proportional to [10, 13, 14]

$$
\alpha(\omega)=\operatorname{Im} \int \boldsymbol{P}^{*}(\boldsymbol{r}, \omega) \boldsymbol{E}(\boldsymbol{r}, \omega) \mathrm{d} \boldsymbol{r},
$$

where $\boldsymbol{P}(\boldsymbol{r}, \omega)$ is the polarization density of the sample, induced by the electric field $\boldsymbol{E}(\boldsymbol{r}, \omega)$. It can be written as

$$
\boldsymbol{P}(\boldsymbol{r}, \omega)=\int \overleftrightarrow{\boldsymbol{\chi}}\left(\boldsymbol{r}, \boldsymbol{r}^{\prime}, \omega\right) \boldsymbol{E}\left(\boldsymbol{r}^{\prime}, \omega\right) \mathrm{d} \boldsymbol{r}^{\prime},
$$

where $\vec{\chi}\left(\boldsymbol{r}, \boldsymbol{r}^{\prime}, \omega\right)$ is the nonlocal susceptibility tensor. In reasonable good quality QWs the amplitude of the confinement energy fluctuations is typically one order of magnitude smaller than the exciton binding energy. In this limit, disorder affects significantly only the center of mass motion through an effective two dimensional potential $V(\boldsymbol{R})[12,15]$, being $\boldsymbol{R} \equiv(x, y)$ the projection of the position vector on the plane of the QW. This effective disorder potential felt by excitons tends to localize the center of mass motion of QW excitons.

In QWs described within the usual envelope-function formalism with isotropic electron and hole dispersions and neglecting fine structure splittings, the susceptibility tensor becomes diagonal with identical elements given by $\mu_{v c}^{2}\left|\phi_{1 s}(0)\right|^{2} \rho(z) \rho\left(z^{\prime}\right) G\left(\boldsymbol{R}, \boldsymbol{R}^{\prime}, \omega\right)$, where $\mu_{v c}$ is the interband bulk dipole moment, $\phi_{1 s}$ describes the relative electron-hole motion (assumed undistorted by disorder), $\rho(z)$ is the product of the electron and hole confinement functions along the growth axis and, finally, the quantity $G\left(\boldsymbol{R}, \boldsymbol{R}^{\prime}, \omega\right)$ is the retarded propagator for the exciton COM motion. The COM propagator obeys the following equation:

$$
\left(\hbar \omega+i \delta-\hat{H}_{\boldsymbol{R}}\right) G\left(\boldsymbol{R}, \boldsymbol{R}^{\prime}, \omega\right)=\delta\left(\boldsymbol{R}-\boldsymbol{R}^{\prime}\right),
$$

where the effective COM Hamiltonian $\hat{H}$ can be written as $\hat{H}_{\boldsymbol{R}}=-\hbar^{2} \nabla^{2} / 2 M+\hbar \omega_{1 s}+V(\boldsymbol{R})$, where $M=m_{e}^{*}+m_{h}^{*}$ is the exciton kinetic mass $\left(m_{e}^{*}\right.$ and $m_{h}^{*}$ are the effective masses of the electron and of the hole) and $\hbar \omega_{1 s}$ is the $1 s$ exciton energy level in the ideal disorder free quantum well.

The effective disorder potential $V(\boldsymbol{R})$ can be modeled as a zero mean, Gauss distributed and spatially correlated process [16] defined by the property $\left\langle V(\boldsymbol{R}) V\left(\boldsymbol{R}^{\prime}\right)\right\rangle=v_{0}^{2} \exp \left(-\left|\boldsymbol{R}-\boldsymbol{R}^{\prime}\right|^{2} / 2 \xi^{2}\right)$, where $\langle\ldots\rangle$ denotes the ensamble average over random conurations; $v_{0}$ is the width of the energy distribution and $\xi$ is the correlation length characterizing the potential fluctuations. Realistic random potentials can be different from this model, which is however widely adopted because it retains the main physical properties in a very simple way. Inserting the above-mentioned expression of $\overleftrightarrow{\chi}$ into Eq. (2), and inserting Eq. (2) into Eq. (1), the total absorbed power in a semiconductor QW can be evaluated according to

$$
\alpha(\omega)=\mu_{v c}^{2}\left|\phi_{1 s}(0)\right|^{2} \operatorname{Im} \int \tilde{E}(\boldsymbol{R}, \omega) G\left(\boldsymbol{R}, \boldsymbol{R}^{\prime}, \omega\right) \tilde{E}\left(\boldsymbol{R}^{\prime}, \omega\right) \mathrm{d} \boldsymbol{R} \mathrm{d} \boldsymbol{R}^{\prime},
$$

where $\tilde{E}(\boldsymbol{R}, \omega)=\int \boldsymbol{E}(\boldsymbol{r}, \omega) \rho(z) \mathrm{d} z$ [we recall that $\boldsymbol{r} \equiv(\boldsymbol{R}, z)$ ]. Considering a light field with a given profile centered around the beam position $\boldsymbol{R}_{0}, \tilde{E}(\boldsymbol{R}, \omega)=E_{0}(\omega) g\left(\boldsymbol{R}-\boldsymbol{R}_{0}\right)$, we may define a local absorption that is a function of the beam position, and relates the total absorbed power to the power of a local excitation (illumination mode),

$$
\alpha_{g}\left(\boldsymbol{R}_{0}, \omega\right) \propto \int G^{I}\left(\boldsymbol{R}, \boldsymbol{R}^{\prime}, \omega\right) g\left(\boldsymbol{R}-\boldsymbol{R}_{0}\right) g\left(\boldsymbol{R}^{\prime}-\boldsymbol{R}_{0}\right) \mathrm{d} \boldsymbol{R} \mathrm{d} \boldsymbol{R}^{\prime},
$$

where $G^{I}$ is the imaginary part of $G$. In the following we describe the eventually confined light beam by a Gaussian EM-profile $g(\boldsymbol{R})=\exp \left[-\left(x^{2}+y^{2}\right) / 2 \sigma^{2}\right]$.

We perform specific calculations for the total absorption under sample illumination, considering both global $(\sigma=\infty)$ and local absorption spectra for different spatial resolutions. Calculations are carried out in real space, mapping on a fine mesh of points the Hamiltonian, which is then tridiagonalized by using the Lanczos algorithm [10, 13]. We adopt an exciton kinetic mass of $m=0.25 m_{0}$ typical for $G a A s / A l G a A s$ quantum wells. The spectra have been calculated by considering a square region of $1 \mu \mathrm{m}^{2}$ which has been divided into $n=300 \times 300$ steps; periodic boundary conditions have been adopted. For all the calculated spectra we used a homogeneous broadening fixed at $\delta=30 \mu \mathrm{eV}$, which could be interpreted as the resolution limit of the spectrometer. 
In order to isolate spectral changes due to excitation of states not accessible by far-field optical probes, we compare far-field absorption spectra (we use $\sigma=\infty$ ) with spatially averaged absorption spectra (Fig. 1). These averaged spectra are composed summing up local spectra obtained centering the confined illuminating beams on a fine mesh of points (with the nearest distance $\Delta_{m} \ll \sigma$ ) on the quantum well plane. Some of the results on GaAs quantum wells are shown in the figure. The obtained global spectrum displays the asymmetric Gaussian shape typical of inhomogeneously broadened exciton lines. The asymmetric shape origins from $k$-vector mixing induced by disorder. We observe that at low spatial resolution (diffraction-limit) the averaged spectra coincide almost perfectly with the far-field spectrum. Increasing the spatial resolution, we find significant spectral changes as blue shift and spectral broadening. Thus we find that at subwavelength resolution, summing up local optical spectra does not reproduce the global spectrum. Although the total excitonic in-plane momentum $K$ is no longer a good quantum number due to disorder, these spectral changes can be understood as a consequence of excitation of high $K$ exciton states not accessible by far-field illumination. We observe that the spectral shape of the low energy region of the spectrum (arising from more localized quantum states) is better maintained at increasing spatial resolutions as compared to the high energy tail.

These results demonstrate that near-field spectroscopy of semiconductor quantum structures does not provide just a spatial selection of inhomogeneous surface systems, in addition it enables the optical detection of states not accessible by far-field optical probes. This ability might be exploited in future experiments on semiconductor quantum structures. It is important to observe that these spectral changes are found at spatially resolutions largely reached by current near-field technology. Recent measurements based on spatially-resolved photoluminescence provided direct information on the spatial and energy distribution of light emitting nanometric centers in narrow QWs [1].

Figure 2 displays numerical results obtained by using the same random potential realization used for all the previous calculations but choosing a larger amplitude $\left(v_{0}=3 \mathrm{meV}\right)$. Comparing Figs. 1 and 2 , it emerges that increasing the disorder amplitude produces a lowering of the discrepancies between far-field and spatially averaged spectra. In particular we observe that the averaged spectrum obtained with FWHM $=47 \mathrm{~nm}$ almost coincides with the far-field spectrum. In contrast the corresponding averaged spectrum in Fig. 1 differs significantly from the far-field spectrum. This result can be interpreted by observing that, increasing disorder, the exciton states suffer from a large degree of localization and the system tends to behave as a system with a local susceptibility.

The numerical calculations displayed in Fig. 1 show that summing up optical spectra with subwavelength spatial resolutions does not reproduce the global spectrum. This demonstrates that spectral

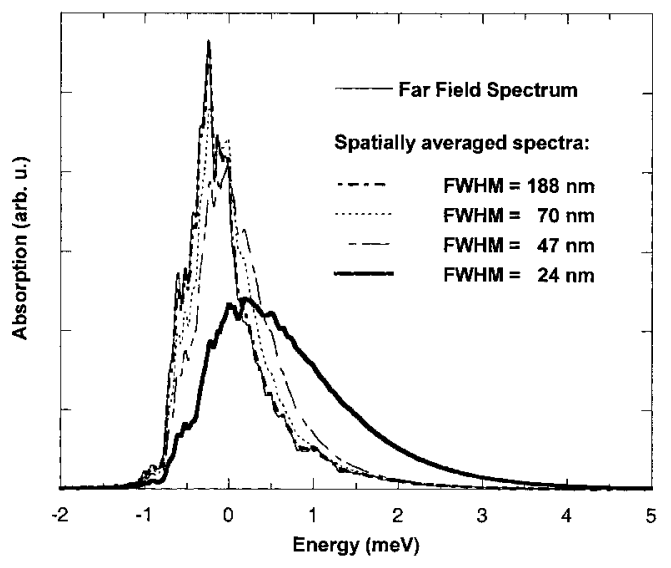

Fig. 1 Far-field absorption spectrum and averaged near-field absorption spectra obtained with different spatial resolutions.

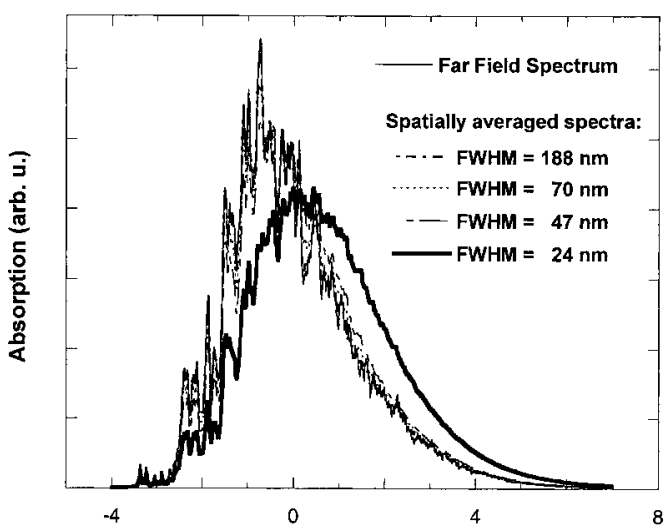

Fig. 2 Far-field absorption spectrum and averaged near-field absorption spectra obtained with the same spatial resolutions of Fig. 1 but choosing a larger amplitude of the random potential $\left(v_{0}=3 \mathrm{meV}\right)$. 
changes at increasing spatial resolutions (due to surface states with high $k$-vectors not accessible by far-field optical excitations) are detectable also in real systems affected by disorder and imperfections. Moreover the spectral changes here described provide a measure of the spatial nonlocality displayed by two-dimensional quantum structures affected by interface fluctuations. Near-field microscopy/spectroscopy of semiconductor quantum structures (due to the nonlocal character of light-matter interaction in these systems) does not provide just a spatial selection of inhomogeneous surface systems, in addition it enables the optical detection of states not accessible by far-field optical probes. This ability could be exploited in future experiments on semiconductor quantum structures.

\section{References}

[1] H. F. Hess, E. Betzig, T. D. Harris, L. N. Pfeiffer, and K. W. West, Science 264, 1740 (1994).

[2] D. Gammon, E. S. Snow, B. V. Shanabrook, D. S. Katzer, and D. Park, Phys. Rev. Lett. 76, 3005 (1996).

[3] D. Gammon, E. S. Snow, B. V. Shanabrook, D. S. Katzer, and D. Park, Science 273, 87 (1996).

[4] J. Hasen, L. N. Pfeiffer, A. Pinczuk, S. He, K. W. West, and B. S. Dennis, Nature (London) 390, 54 (1997).

[5] J. R. Guest, T. H. Stievater, Gang Chen, E. A. Tabak, B. G. Orr, D. G. Steel, D. Gammon, and D. S. Katzer, Science 293, 2224 (2001).

[6] S. Savasta, G. Martino, and R. Girlanda, Phys. Rev. B 61, 13852 (2000).

[7] A. V. Shchegrov, K. Joulain, R. Carminati, and J. J. Greffet, Phys. Rev. Lett. 85, 1548 (2000).

[8] G. Pistone, S. Savasta, O. Di Stefano, and R. Girlanda, Phys. Rev. B, to appear.

[9] E. Runge and R. Zimmermann, phys. stat. sol. (b) 206, 167 (1998).

[10] O. Di Stefano, S. Savasta, G. Martino, and R. Girlanda, Appl. Phys. Lett. 77, 2804 (2000).

[11] C. Weisbuch, R. Dingle, A.C. Grossard, and W. Wiegmann, Solid State Commun. 206, 219 (1981).

[12] R. Zimmermann, phys. stat. sol. (b) 173, 129 (1992).

[13] O. Di Stefano, S. Savasta, G. Martino, and R. Girlanda, Phys. Rev. B 62, 11071 (2000).

[14] O. Mauritz, G. Goldoni, F. Rossi, and E. Molinari, Phys. Rev. Lett. 82, 847 (1999).

[15] S. D. Baranovskii, U. Doerr, P. Thomas, A. Naumov, and W. Gebhardt, Phys. Rev. B 48, 17149 (1993).

[16] S. Glutsch, D. S. Chemla, and F. Bechstedt, Phys. Rev. B 54, 11592 (1996). 